\title{
DETERMINAÇÃO DE METIL-ETIL-CETONA EM AMOSTRAS DE URINA COM AMOSTRAGEM POR MICRO EXTRAÇÃO EM FASE SÓLIDA (MEFS) EM HEADSPACE ASSOCIADA À CROMATOGRAFIA GASOSA COM DETECTOR DE IONIZAÇÃO DE CHAMA (CG-DIC)
}

\author{
Marina Venzon Antunes e Rafael Linden* \\ Instituto de Ciências da Saúde, Centro Universitário Feevale, Rodovia RS 239, n. 2755, 93352-000 Novo Hamburgo - RS, \\ Brasil
}

Recebido em1/2/08; aceito em 20/6/08; publicado na web em 24/10/08

\begin{abstract}
DETERMINATION OF METHYL ETHYL KETONE IN URINE SAMPLES BY HEADSPACE SOLID PHASE MICRO EXTRACTION (SPME) SAMPLING ASSOCIATED TO GAS CHROMATOGRAPHY WITH FLAME-IONIZATION DETECTOR (GC-FID). Methyl ethyl ketone (MEK) is a solvent commonly used in chemical, paint and shoe industry. The aim of this study was to develop and validate a method for urinary quantification of MEK, employing headspace solid phase micro extraction sampling (SPME) coupled to gas chromatography with flame ionization detection (GC-FID). The calibration curve $(\mathrm{y}=4.6851 \mathrm{x}-0.0011)$ presented good linearity with $\mathrm{r}^{2}=0.9993$. Accuracy (94-109\%), intra-assay precision (4.07-5.91\%) and inter-assay precision (3.03$5.62 \%$ ) were acceptable. The quantification limit was $0.19 \mathrm{mg} / \mathrm{L}$. This low cost method can be used routinely in the biological monitoring of occupational exposure to MEK, according to the requirements of the Brazilian legislation.
\end{abstract}

Keywords: methyl ethyl ketone; SPME-GC-FID; biological monitoring.

\section{INTRODUÇÃO}

A metil-etil-cetona (MEC) é amplamente utilizada na indústria calçadista, moveleira, química, de tintas, beneficiamento de madeira, entre outras. Também é utilizada como solvente em removedores de tinta, adesivos e revestimentos poliméricos. ${ }^{1,2} \mathrm{~A}$ MEC também é denominada 2-butanona e metil acetona, tem peso molecular de 72,11 u.m.a. e densidade $0,805 \mathrm{~g} / \mathrm{mL} .^{2} \mathrm{Na}$ exposição ocupacional, as principais vias de introdução da MEC são a pulmonar e cutânea, destacando-se a primeira. ${ }^{3,4}$

A exposição crônica à MEC pode causar neurotoxicidade periférica e central sendo, portanto, recomendado o monitoramento biológico dos indivíduos expostos ocupacionalmente a este composto. ${ }^{4}$ Devido à elevada correlação entre os níveis urinários de MEC e a exposição ambiental, a determinação das suas concentrações urinárias é utilizada no monitoramento da exposição ocupacional. ${ }^{5}$

No Brasil, a Norma Regulamentadora No 7 (NR-7) do Ministério do Trabalho e Emprego estabelece a obrigatoriedade, por parte das empresas e instituições que possuam trabalhadores expostos à MEC, do monitoramento biológico periódico destes trabalhadores. ${ }^{6}$ A NR-7 determina a quantificação da MEC em amostras de urina de final de jornada, com Índice Biológico Máximo Permitido (IBMP) de $2 \mathrm{mg} / \mathrm{L}$.

Diversos métodos analíticos para determinação de MEC em urina estão descritos na literatura, empregando extração com solventes. ${ }^{7-9}$ Alternativamente, Pellizari et al. apresentaram uma técnica com amostragem por headspace e quantificação por cromatografia gasosa com detector seletivo de massas (CG-EM). ${ }^{10}$

Uma alternativa mais recente é o emprego de microextração em fase sólida (MEFS), que apresenta como vantagens a inexigibilidade do uso de solventes orgânicos e a possibilidade de automação. Esta possibilidade foi explorada por Chou et al. ${ }^{1}$ para a determinação de MEC em amostras de urina, com procedimento de MEFS manual.

O presente trabalho descreve um método simples e automatizado

*e-mail: rafael.linden@feevale.br para a determinação de MEC em amostras de urina, com propósito de monitoramento biológico da exposição a este solvente, empregando MEFS automatizada associada à cromatografia gasosa com detector de ionização de chama (CG-DIC).

\section{PARTE EXPERIMENTAL}

\section{Reagentes e materiais}

A metil-etil-cetona (99\%) foi obtida da Mallinckrodt Chemicals (Phillipsburg, USA). O cloreto de sódio foi obtido da Nuclear (São Paulo, Brasil). A fibra para MEFS revestida de polidimetilsiloxano (PDMS), com espessura do filme de $100 \mu \mathrm{m}$, foi proveniente da Supelco (Bellefonte, USA). A fibra foi condicionada através da sua inserção no injetor do cromatógrafo aquecido a $250^{\circ} \mathrm{C}$, sendo mantida nesta condição por $30 \mathrm{~min}$, conforme instruções do fabricante.

\section{Equipamentos}

Todo o sistema de cromatografia gasosa foi proveniente da Varian (Midlesburg, Holanda). O procedimento de MEFS foi realizado em um autoamostrador Varian CP-8410, com bandeja para recirculação de água. A temperatura das amostras foi controlada através de banhomaria com circulação de água Nova Ética (Vargem Grande Paulista, Brasil). As análises foram realizadas em cromatógrafo gasoso Varian CP-3800, com detector de ionização de chama. O sistema cromatográfico foi controlado pelo programa Galaxie. A coluna capilar utilizada na separação cromatográfica foi CP-WAX 52 CB da Varian (Midlesburg, Holanda), com $30 \mathrm{~m}$ de comprimento, 0,25 mm de diâmetro interno e revestimento interno com espessura de $0,25 \mu \mathrm{m}$.

\section{Condições cromatográficas}

A temperatura do injetor, operado na condição splitless, foi 280 ${ }^{\circ} \mathrm{C}$. A coluna foi submetida à seguinte programação de temperatura: $50{ }^{\circ} \mathrm{C}$ por $1 \mathrm{~min}$, rampa de $20^{\circ} \mathrm{C}$ por min até $150{ }^{\circ} \mathrm{C}$ e $1 \mathrm{~min}$ a 150 
${ }^{\circ} \mathrm{C}$. Hélio foi utilizado como gás de arraste, com fluxo constante de $2,5 \mathrm{~mL} / \mathrm{min}$. $\mathrm{O}$ detector foi mantido a $250{ }^{\circ} \mathrm{C}$.

\section{Coleta e conservação das amostras}

Foram obtidas amostras de urina de trabalhadores expostos à MEC em uma indústria química de tintas para calçados $(n=15)$. Todas as amostras foram coletadas no $4^{\circ}$ dia útil da semana, no final da jornada de trabalho. Imediatamente após a coleta, as amostras foram armazenadas sob refrigeração a $4{ }^{\circ} \mathrm{C}$. As análises foram realizadas no dia seguinte à coleta.

\section{Otimização da MEFS}

As condições experimentais para a MEFS foram selecionadas através de metodologia de superfície de resposta, que é definida como um conjunto de técnicas matemáticas e estatísticas empregadas para modelar e analisar problemas nos quais uma variável de resposta é influenciada por diversas variáveis operacionais com o objetivo de otimizar esta resposta. ${ }^{11}$ Através de um desenho experimental adequado é possível obter uma equação de regressão de $2^{\text {a }}$ ordem que descreve a relação entre as variáveis do processo e a variável de resposta. Foi selecionado um desenho experimental do tipo Box-Behnken, com as seguintes variáveis experimentais: temperatura das amostras (40, 50 e $\left.60{ }^{\circ} \mathrm{C}\right)$, concentração de cloreto de sódio na amostra $(0,15 ; 0,25$ e $0,35 \mathrm{~g} / \mathrm{mL}$ ) e tempo de exposição da fibra durante a etapa de adsorção (5; 17,5 e $30 \mathrm{~min}$ ), com um total de 17 experimentos (Tabela 1). Os cálculos foram realizados utilizando o programa Design Expert ${ }^{\circledR} 7.0$ da Stat-Ease (Minneapolis, EUA).

\section{Preparo das amostras}

A preparação das amostras pela técnica de MEFS por headspace seguiu as condições determinadas após o experimento de otimização. Em tubo de ensaio com tampa rosca, adicionou-se uma alíquota de $750 \mu \mathrm{L}$ de urina e $0,2625 \mathrm{~g}$ de cloreto de sódio, que foram homogeneizados em agitador vórtex por $30 \mathrm{~s}$. A amostra foi transferida para vial de $2 \mathrm{~mL}$ e colocada em autoamostrador com temperatura mantida a $60^{\circ} \mathrm{C}$. A fibra de MEFS permaneceu exposta ao headspace da amostra por $30 \mathrm{~min}$. A dessorção foi realizada por exposição da fibra ao injetor do cromatógrafo por $3 \mathrm{~min}$.

\section{Avaliação da seletividade}

Amostras de urina de 6 indivíduos não expostos à metil-etil-cetona foram processadas e a presença de picos interferentes foi avaliada. Também foram analisadas amostras brancas de urina adicionadas de acetona, etanol, metanol, tolueno e xileno, na concentração de $5 \mathrm{mg} / \mathrm{L}$ para avaliação de potenciais interferentes.

\section{Curva de calibração}

Para a construção da curva de calibração foi utilizada a média de 4 determinações de cada calibrador de MEC, nas concentrações de 0,$327 ; 0,633 ; 1,265 ; 2,53 ; 5,06$ e $10,12 \mathrm{mg} / \mathrm{L}$. Os calibradores foram obtidos a partir da diluição com urina isenta do analito de uma solução-mãe de MEC a 1,012 g/L, preparada em água purificada. A curva de calibração foi construída através de regressão linear pelo método dos mínimos quadrados, estabelecendo a correlação entre a área dos picos referentes a MEC (y) e as concentrações adicionadas (x). A curva foi avaliada com base no coeficiente de determinação $\left(\mathrm{r}^{2}\right)$. A equação de regressão foi obtida empregando o programa Microsoft Excel ${ }^{\circledR}$.

\section{Avaliação do efeito de memória da fibra de MEFS}

Foram processadas amostras de urina contendo MEC na concentração de $20,24 \mathrm{mg} / \mathrm{L}$, sucedidas por amostras de urina isentas do analito, em triplicata. O efeito de memória foi avaliado através da determinação de MEC nas amostras brancas.

\section{Ensaios de precisão e exatidão}

A precisão e a exatidão do método foram avaliadas através de análises em três níveis de concentração para cada analito, realizadas em triplicata e repetidas em 5 dias diferentes. Os calibradores utilizados foram preparados em água purificada. As precisões intra-ensaios e inter-ensaios foram calculadas pela análise de variância (ANOVA), usando dia como variável agrupadora. A exatidão foi calculada como percentagem média obtida do valor teórico adicionado na amostra. As concentrações utilizadas no estudo de precisão e exatidão foram 0,662; 2,07 e 6,48 mg/L.

\section{Estabilidade}

Amostras de urina adicionadas de MEC na concentração de 2,07 mg/L foram preparadas e imediatamente armazenadas a $4{ }^{\circ} \mathrm{C}$. Alíquotas destas amostras foram analisadas no dia da preparação e 2, 7 e 14 dias após. A estabilidade foi avaliada através do C.V \% dos valores obtidos entre os valores de concentração de MEC calculados através da curva de calibração.

\section{Limites de detecção e quantificação}

Os limites de detecção e quantificação de cada analito foram determinados a partir do cálculo da concentração de 3 e 10 vezes, respectivamente, a área do ruído da linha de base produzido pela matriz.

\section{Controle de qualidade}

Em cada lote de análises, composto de no máximo 20 amostras, uma amostra branca foi analisada no início da rotina e um calibrador, com concentração de $2,0 \mathrm{mg} / \mathrm{L}$, foi analisado no início e no final desta seqüência.

\section{RESULTADOS E DISCUSSÃO}

\section{Otimização da MEFS}

A técnica de MEFS apresenta como principais vantagens a inexigibilidade de solventes orgânicos e a possibilidade de automação, o que pode contribuir para obtenção de métodos analíticos com maior segurança para os técnicos e o meio ambiente, com elevada produtividade. Entretanto, a determinação das condições experimentais mais adequadas apresenta-se como um problema multifatorial, que pode ser abordado por estratégias de tentativa e erro ou, alternativamente, através de experimentos estatisticamente planejados. A segunda abordagem em geral é mais eficiente, minimizando o número de experimentos, e permite modelar matematicamente os processos estudados, permitindo o cálculo de condições otimizadas. Neste estudo, a otimização da MEFS foi realizada através de um desenho experimental do tipo Box-Behnken devido a sua economicidade e pela possibilidade de modelar superfícies de resposta. As variáveis estudadas foram concentração do agente de salting out (cloreto de sódio), temperatura da amostra e tempo de exposição da fibra de MEFS ao headspace da amostra. A variável otimizada foi a área do pico de MEC. Os níveis das variáveis estudadas e os resultados obtidos para área do pico de metil-etil-cetona são apresentados na Tabela 1. 
Tabela 1. Desenho experimental de otimização da MEFS

\begin{tabular}{lcccc}
\hline Experimento & \% NaCl & $\begin{array}{c}\text { Tempo de ex- } \\
\text { posição (min) }\end{array}$ & $\begin{array}{c}\text { Temperatura } \\
\left({ }^{\circ} \mathrm{C}\right)\end{array}$ & $\begin{array}{c}\text { Área do pico } \\
\text { de MEC } \\
(\mu \mathrm{V} \text { min })\end{array}$ \\
\hline 1 & 0,15 & 17,5 & 60,0 & 11.3 \\
2 & 0,25 & 30,0 & 60,0 & 13.4 \\
3 & 0,35 & 30,0 & 50,0 & 13.7 \\
4 & 0,25 & 5,0 & 60,0 & 10.7 \\
5 & 0,15 & 5,0 & 50,0 & 7.4 \\
6 & 0,35 & 17,5 & 60,0 & 12.8 \\
7 & 0,25 & 17,5 & 50,0 & 11.9 \\
8 & 0,25 & 17,5 & 50,0 & 10.2 \\
9 & 0,15 & 30,0 & 50,0 & 8.3 \\
10 & 0,25 & 17,5 & 50,0 & 10.2 \\
11 & 0,35 & 17,5 & 40,0 & 12.4 \\
12 & 0,25 & 5,0 & 40,0 & 8,0 \\
13 & 0,25 & 17,5 & 50,0 & 11.8 \\
14 & 0,35 & 5,0 & 50,0 & 10.7 \\
15 & 0,25 & 17,5 & 50,0 & 12.7 \\
16 & 0,15 & 17,5 & 40,0 & 7.9 \\
17 & 0,25 & 30,0 & 40,0 & 11.6 \\
\hline
\end{tabular}

As condições otimizadas foram avaliadas visualmente através de gráficos de contornos (Figura 1) e calculadas através do programa Design Expert ${ }^{\circledR}$.

A resposta foi modelada através da Equação 1. O modelo ajustado para os dados experimentais, avaliado através de análise de variância (ANOVA), apresentou adequação de ajuste significante e falta-deajuste não significante $(\mathrm{P}>0,05)$.

Área pico $=-0.68390+18.37500 *$ Concentração $\mathrm{NaCl}+0.10200$

* Tempo $+0.10375 *$ Temperatura

Considerando a adequação do modelo, foi possível calcular condições operacionais otimizadas com base na Equação 1. Dentre as três variáveis estudadas, apenas a concentração de cloreto de sódio não foi selecionada em seu nível máximo. A eficiência da extração cresceu com o aumento da temperatura da amostra e com o tempo de exposição, as quais foram selecionadas em seus níveis superiores. Foi calculada uma solução para maximizar a resposta área do pico de MEC, limitando o tempo de exposição da fibra. Entretanto, a resposta otimizada somente foi possível com tempo de 30 min e esta condição foi selecionada com o propósito de obter a maior sensibilidade possível. A condição otimizada, selecionada para a obtenção da curva de calibração e para os demais ensaios de validação, empregou 0,2625 $\mathrm{g}$ de cloreto de sódio em $750 \mu \mathrm{L}$ de urina, temperatura do autoamostrador de $60{ }^{\circ} \mathrm{C}$ e tempo de exposição da fibra de $30 \mathrm{~min}$.

A automação da MEFS se valeu de um autoamostrador CP-8400 modificado através da adaptação, à bandeja de posicionamento das amostras, de um de banho-maria com circulação de água. Esta modificação permitiu controle de todas as variáveis importantes da MEFS em um sistema de baixo custo, em comparação com os robôs de automação de MEFS disponíveis no mercado.

\section{Condições cromatográficas e parâmetros de validação}

O tempo total da análise cromatográfica foi de $7 \mathrm{~min}$. O tempo médio de retenção da MEC foi de 1,83 min. A Figura 2 apresenta cromatogramas típicos obtidos com a metodologia nos quais pode-se observar a ausência
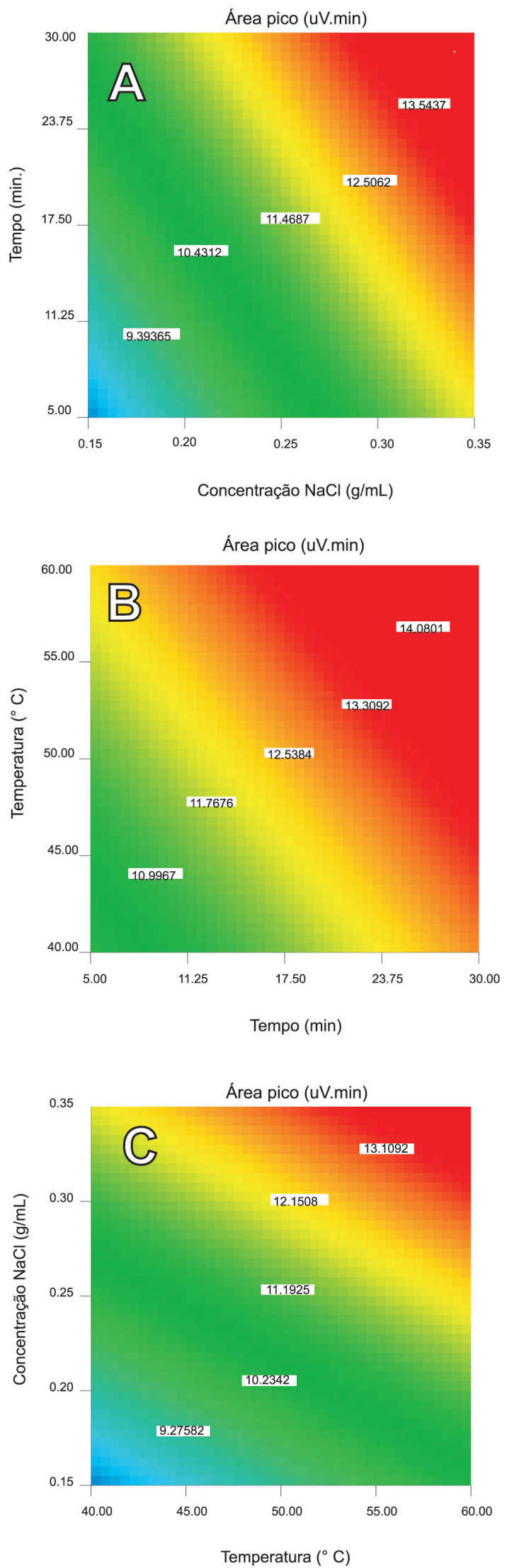

Figura 1. Gráficos de contorno da resposta da área do pico de $M E C(\mu V$. $\mathrm{min}$ ): (A) relação tempo de absorção ( $\mathrm{min})$ x concentração de $\mathrm{NaCl}(\mathrm{g} / \mathrm{mL})$; (B) relação temperatura autoamostrador $\left({ }^{\circ} \mathrm{C}\right)$ x tempo (min); (C) relação concentração de $\mathrm{NaCl}(\mathrm{g} / \mathrm{mL})$ x temperatura autoamostrador $\left({ }^{\circ} \mathrm{C}\right)$ 
de picos interferentes no tempo de retenção relativo à MEC na amostra de indivíduo não exposto a esta substância. Também foi observada a ausência de interferentes nos estudos de seletividade com amostras fornecidas por voluntários. A presença de acetona, metanol, etanol, tolueno e xileno, com tempos de retenção de 1,$49 ; 1,75 ; 1,93 ; 2,59$ e $3,38 \mathrm{~min}$, respectivamente, também não interferiu na quantificação de MEC.
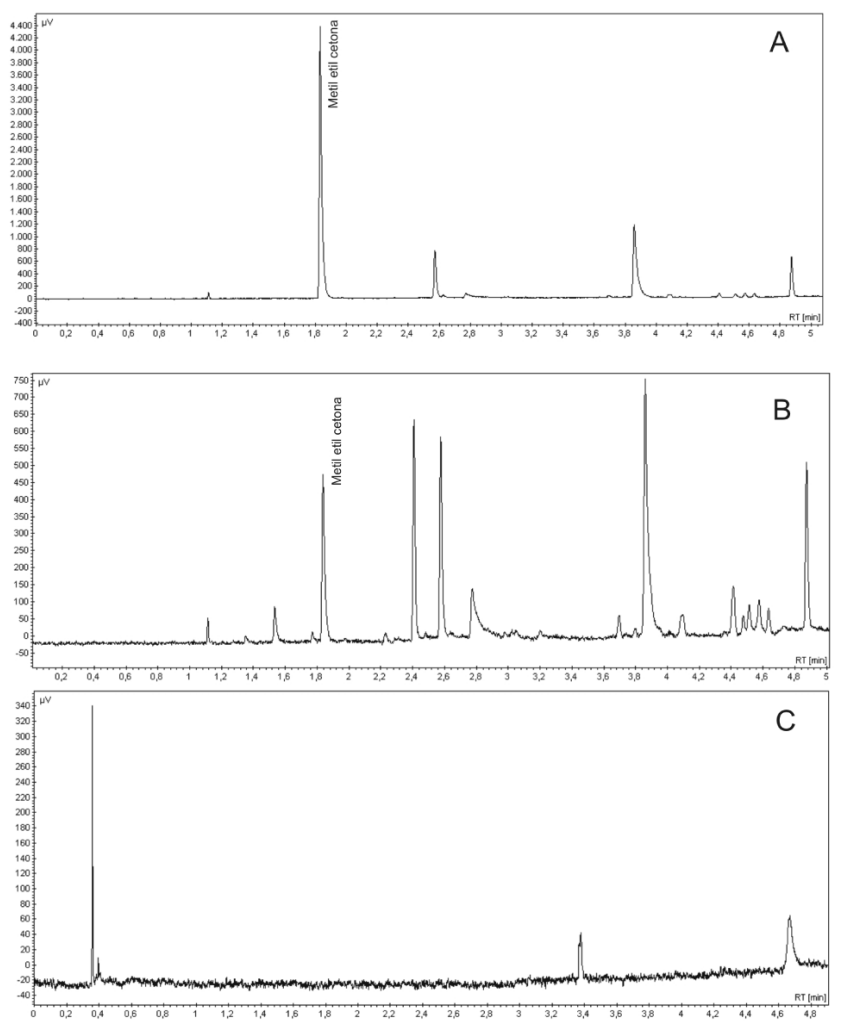

Figura 2. Separação cromatográfica: (A) calibrador contendo MEC (20,50 $\mathrm{mg} / \mathrm{L}) ;(B)$ amostra de urina de trabalhador exposto à $M E C(2,18 \mathrm{mg} / \mathrm{L}) ;(C)$ amostra de urina de indivíduo não exposto à MEC

A curva de calibração, expressa pela função y $=4,6851 \mathrm{x}-0,0011$, apresentou adequada linearidade, com coeficiente de determinação $\left(\mathrm{r}^{2}\right)$ de 0,9999 . Os valores quantitativos obtidos não foram influenciados por eventual efeito de memória da fibra de MEFS, visto que amostras de urina isentas de MEC não apresentaram picos detectáveis deste analito quando processadas imediatamente após amostras com concentrações duas vezes superiores à do maior calibrador da curva. Considerando a elevada repetibilidade do procedimento de MEFS proporcionado pela automação, foi possível obter determinações quantitativas com elevada precisão e exatidão, conforme dados obtidos nos experimentos de validação e apresentados na Tabela 2 . Os valores obtidos de precisão e exatidão encontram-se dentro dos critérios de aceitação para métodos bioanalíticos preconizados por Shah et al.. ${ }^{12}$ As concentrações de MEC mostraram-se estáveis quando as amostras de urina foram mantidas em refrigeração, em um intervalo de até 14 dias, com C.V \% entre os valores pré e pós-armazenamento de 3,98\%. Para evitar variações devido à redução da capacidade absortiva da fibra de MEFS, amostras-controle foram processadas em cada conjunto de amostras-teste, conforme descrito no item controle de qualidade. Em mais de uma centena de amostras processadas, nenhum teste foi rejeitado devido a variações inaceitáveis dos valores-alvo dos controles. Os limites de detecção e quantificação foram 0,057 e $0,19 \mathrm{mg} / \mathrm{L}$, respectivamente. Considerando o propósito da metodologia proposta neste estudo, focada no monitoramento biológico e com valores limiares definidos pela NR-7, os limites de detecção e
Tabela 2. Parâmetros de validação do método

\begin{tabular}{lccc}
\hline $\begin{array}{l}\text { Concentração } \\
(\mathrm{mg} / \mathrm{L})\end{array}$ & $\begin{array}{c}\text { Intra-dias } \\
\text { (C.V. \%) }\end{array}$ & $\begin{array}{c}\text { Inter-dias } \\
\text { (C.V. \%) }\end{array}$ & Exatidão(\%) \\
\hline 0,662 & 4,91 & 4,62 & 109 \\
2,07 & 5,60 & 3,55 & 101 \\
6,48 & 4,67 & 3,27 & 94 \\
\hline
\end{tabular}

quantificação são bastante inferiores ao IBMP de 2,0 mg/L.

Na Tabela 3 são apresentados os intervalos das concentrações obtidas através das análises das amostras de urina dos indivíduos expostos ocupacionalmente à MEC $(\mathrm{n}=15)$. As concentrações estão de acordo com os achados de Petrarulo e Pellegrino, ${ }^{9}$ que obtiveram valores entre 0,3 a 3,6 mg/L $(\mathrm{n}=60)$, para amostras de indivíduos expostos coletadas no final da jornada de trabalho. Dentre os 15 indivíduos avaliados no presente estudo, 3 apresentaram valores acima do IBMP, com concentrações de 2,18; 3,71 e 3,21 mg/L.

Tabela 3. Resumos das análises realizadas em urina de indivíduos expostos ocupacionalmente à metil etil cetona

\begin{tabular}{lcc}
\hline Número de Indivíduos & Faixa de valores & $\begin{array}{c}\text { Média } \pm \text { desvio } \\
\text { padrão }\end{array}$ \\
\hline 15 & $0,21-3,71 \mathrm{mg} / \mathrm{L}$ & $1,4 \pm 1,09 \mathrm{mg} / \mathrm{L}$ \\
\hline
\end{tabular}

\section{CONCLUSÃO}

Um método para a determinação de metil-etil-cetona por MEFSCG-DIC foi desenvolvido e validado. Os parâmetros precisão, exatidão e sensibilidade são adequados para o monitoramento biológico da MEC. O método emprega um sistema de automação modificado para permitir o controle da temperatura das amostras. O método foi aplicado no monitoramento biológico de trabalhadores expostos ocupacionalmente a esse solvente e mostrou-se apropriado a esta finalidade.

\section{REFERÊNCIAS}

1. Chou, J. S.; Shih, T. S.; Chen, C. M.; J. Occup. Environ. Med. 1999, 41, 1042 .

2. United States Environmental Protection Agency; Toxicological review of methyl ethyl ketone (EPA 635/R-03/009), EPA: Washington, 2003.

3. Oga, S.; Fundamentos de toxicologia, $2^{\mathrm{a}}$ ed., Atheneu: São Paulo, 2003.

4. Xiao, J. Q.; Levin, S. M.; Am. J. Ind. Med. 2000, 37, 44.

5. Imbriani, M.; Ghittori, S.; Int. Arch. Occup. Environ. Health 2005, 78, 1.

6. http://www.mte.gov.br/legislacao/normas_regulamentadoras/nr_07.asp, acessada em Dezembro 2007.

7. Kezic, S.; Monster, A. C.; J. Chromatogr. 1988, 148, 275.

8. Van Doorn, J. E.; De Cock, J.; Kezic, S.; Monster, A. C.; J. Chromatogr. 1989, 489, 419.

9. Petrarulo, M.; Pellegrino, S.; J. Chromatogr., B: Anal. Technol. Biomed. Life Sci. 1992, 579, 324.

10. Miyasaka, M.; Kumai, M.; Koizumi, A.; Watanabe, T.; Kurasako, K.; Sato, K.; Ikeda, M.; Int. Arch. Occup. Environ. Health 1982, 50, 131.

11. Myers, R. H.; Montgomery, D. C.; Response Surface Methodology, $2^{\text {nd }}$ ed. ,Wiley-Interscience: New York, 2002.

12. Shah, V. P.; Midha, K. K.; Findlay, J. W.; Hill, H. M.; Hulse, J. D.; McGilveray, I. J.; McKay, G.; Miller, K. J.; Patnaik, R. N.; Powell, M. L.; Tonelli, A; Viswanathan, C. T.; Yacobi, A.; Pharm. Res. 2000, 17, 1551. 